\title{
The relevancy of controlled nanocrystallization on rifampicin characteristics and cytotoxicity
}

\author{
This article was published in the following Dove Press journal: \\ International Journal of Nanomedicine \\ 19 May 2016 \\ Number of times this article has been viewed
}

\author{
Salma M Mohyeldin \\ Mohammed M Mehanna \\ Nazik A Elgindy \\ Department of Industrial Pharmacy, \\ Faculty of Pharmacy, Alexandria \\ University, Alexandria, Egypt
}

Purpose: This article investigated the influence of novel rifampicin nanosuspension (RIF NS) for enhancing drug delivery properties.

Methods: RIF NS was fabricated using the antisolvent precipitation technique. The impact of solvent type and flow rate, stabilizer type and concentration, and stirring time and apparatus together with the solvent-antisolvent volume ratio on its controlled nanocrystallization has been evaluated. NSs were characterized by transmission electron microscopy, particle size and zeta potential analysis, solubility, and dissolution profiles. The compatibility between RIF and the stabilizer was investigated via Fourier transform infrared spectroscopy and the differential scanning calorimetry techniques. The shelf-life stability of the RIF NS was assessed within a period of 3 months at different storage temperatures. Cell cytotoxicity was evaluated using 3(4,5-dimethylthiazol-2-yl)-2,5-diphenyltetrazolium bromide (MTT) assay on lung epithelial cells.

Results: Polyvinyl alcohol at $0.4 \% \mathrm{w} / \mathrm{v}, 1: 15$ methanol to deionized water volume ratio and 30-minutes sonication were the optimal parameters for RIF NS preparation. Nanocrystals were obtained with a nanometeric particle size $(101 \mathrm{~nm})$ and a negative zeta potential $(-26 \mathrm{mV})$. NS exhibited a 50-fold enhancement in RIF solubility and 97\% of RIF was dissolved after 10 minutes. The RIF NS was stable at $4 \pm 0.5^{\circ} \mathrm{C}$ with no significant change in particle size or zeta potential. The MTT cytotoxicity assay of RIF NS demonstrated a good safety profile and reduction in cell cytotoxicity with half maximal inhibitory concentration values of 0.5 and $0.8 \mathrm{mg} / \mathrm{mL}$ for free RIF and RIF NS, respectively.

Conclusion: A novel RIF NS could be followed as an approach for enhancing RIF physicochemical characteristics with a prominence of a safer and better drug delivery.

Keywords: controlled nanocrystallization, cytotoxicity, nanosuspension, polyvinyl alcohol, rifampicin

\section{Introduction}

Tuberculosis (TB) is a deadly and persistent respiratory infection that is considered to be one of the main provocations in the public health. ${ }^{1}$ According to the World Health Organization reports, TB together with the acquired immunodeficiency syndrome and malaria are the most fatal diseases worldwide; therefore, better management must be assessed. ${ }^{1}$ The current TB therapy possesses many obstacles as the oral drug administration requires a prolonged time over 6-9 months resulting in a poor patient compliance. ${ }^{2}$ For decades, TB is treated by first-line anti-TB drugs regimen, which include isoniazid, rifampicin (RIF), pyrazinamide, and ethambutol. ${ }^{2}$ In some cases, multidrug-resistant TB has been determined as a consequence of bacterial resistance to one or more anti-TB first-line drugs. Treatment of such cases is much more difficult; the therapy is based on second-line agents that include amino glycoside antibiotics, cycloserine, ethionamide, and fluoroquinolones. ${ }^{1,2}$ Conversely, these agents are more

\footnotetext{
Correspondence: Mohammed M Mehanna

Department of Industrial Pharmacy,

Faculty of Pharmacy, Alexandria

University, El Sultan Hussein St.,

Alexandria, 21521, Egypt

Tel +2031003409504

Emaildr_mehanna@yahoo.com
}

(c) (1) (5) ( 2016 Mohyeldin et al. This work is published and licensed by Dove Medical Press Limited. The full terms of this license are available at https://www.dovepress.com/terms.php cC. hereby accept the Terms. Non-commercial uses of the work are permitted without any further permission from Dove Medical Press Limited, provided the work is properly attributed. For permission for commercial use of this work, please see paragraphs 4.2 and 5 of our Terms (https://www.dovepress.com/terms.php). 
toxic, more expensive, and less effective than the standard first-line therapy. Moreover, the second-line therapy requires a long duration up to 9 months or 1 year to be sure of a complete cure in such cases. ${ }^{3}$

Despite being the most effective antitubercular drug, challenges of RIF bioavailability still exist. One of these challenges is that its therapeutic activity is based on its concentration at the target site. ${ }^{4}$ Since RIF is a Biopharmaceutics Classification System class II member possessing low aqueous solubility and high permeability, its dissolution rate and hence bioavailability are limited. ${ }^{5}$ However, a recent report revealed the low permeability of RIF through the intestinal cell, proposing a new classification for the drug to class IV. ${ }^{6}$ In addition, oral administration of RIF causes undesirable side effects, including nausea, flu-like syndrome associated with acute renal failure, hepatotoxicity, and agranulocytosis. ${ }^{7}$ To circumvent RIF drawbacks, various investigations have been reported for solubilizing, delivering high concentration of the drug directly, and sustainably at the site of infection. ${ }^{8,9}$ Other attempts were carried out to increase the RIF solubility involving the complexation with different cyclodextrin derivatives and altering its crystal structure and particle morphology by a mean of a polymorphic transformation into a flake-like crystal hydrate. ${ }^{10,11}$

Over recent years, nanosuspension (NS) technology received a great interest in overcoming the obstacles of poorly soluble drugs. NSs are colloidal dispersions of the pure drug in an outer liquid phase with particle size ranging between 100 and $1,000 \mathrm{~nm} .{ }^{12}$ Several merits were accompanied with this nanodispersion, namely, improved saturation solubility, accelerated dissolution rate, and increased stability of some drugs. Moreover, the large surface area as a result of particle size reduction enhances the solubility as well as the bioavailability of poorly soluble drugs as described by the Ostwald-Freundlich equation. ${ }^{13}$ Currently, NSs have been widely reported as a drug delivery system for different routes of administration, such as parenteral delivery of p-terphenyl derivative, ${ }^{14}$ oral delivery of diosmin, ${ }^{15}$ ocular delivery of amphotericin, ${ }^{16}$ and intranasal delivery of carvedilol. ${ }^{17}$

Generally, there are two main approaches for preparing NSs; top down and bottom up technology. ${ }^{12}$ The bottom up technology involves building particles up from the molecular state as in the antisolvent precipitation process. ${ }^{18}$ This technology is a popular approach used for its simplicity and low setup cost. ${ }^{18}$ It has been widely reported in many studies for preparing different drug nanocarriers as nitrendipine, ${ }^{19}$ quercetin, ${ }^{20}$ amoitone $\mathrm{B},{ }^{21}$ and camptothecin. ${ }^{22}$

Realizing the obstacles of RIF, the impact of NS on enhancing its water solubility as well as dissolution characteristics will be a field of interest. Accordingly, the aim of the current work is to fabricate a novel RIF NS utilizing the simple antisolvent precipitation method in the presence of different stabilizers. The influence of several preparative variables has been investigated in order to control and optimize the process. In vitro characterization of RIF NS was assessed by dynamic light scattering, transmission electron microscopy (TEM), solubility, and dissolution testing. The interaction between RIF and polymers in the NS was investigated using Fourier transform infrared spectroscopy and differential scanning calorimetry (DSC) techniques. Furthermore, NS physical stability and in vitro cytotoxicity were evaluated in comparison to free RIF.

\section{Materials and methods \\ Materials}

Ethical approval was not sought from the IRB of Alexandria University because the study involves no human or animal. According to our regulations, no IRB is required for cell line studies. RIF was obtained as a gift sample from Medical Union Pharmaceuticals Company, Alexandria, Egypt. Poloxamer 188 and 407 were purchased from Sigma-Aldrich Co. (St Louis, MO, USA). Soya phosphatidyl choline (SPC75) was provided by Lipoid Company, Ludwigshafen, Germany. Hydroxy propyl methylcellulose (HPMC), viscosity of 15 and 4,000 cp, was a kind gift from Borg Pharmaceutical Ind., Alexandria, Egypt. Tween 80, polyvinyl alcohol (PVA, molecular weight [MW] 194) and sodium lauryl sulfate (SLS) were purchased from Adwic, El-Nasr Pharmaceutical Co., Alexandria, Egypt. Polyvinylpyrrolidone k30 (PVP) was received as a gift from Medizen Pharmaceutical Industries, Alexandria, Egypt. All organic solvents (methanol, ethanol, and isopropyl alcohol) and propylene glycol were of analytical grade and purchased from El-Nasr Pharmaceutical Co.

\section{Selection of RIF organic solvent}

RIF solubility in various organic solvents was determined in order to select the appropriate solvent to be used during NS preparation. Saturation solubility in each solvent was experimentally established following Higuchi and Connors method. ${ }^{23}$ Excess amount of free RIF were added to $5 \mathrm{~mL}$ of different solvents in capped amber glass vials. These vials were tightly closed and shaken for 24 hours at $37 \pm 0.5^{\circ} \mathrm{C}$ in a thermostatically controlled water bath (GFL, type 1083, GFL Gesellschaft für Labortechnik mbH, Burgwedel, Germany). The dispersions were left for equilibrium for another 24 hours then filtered through $0.45 \mu \mathrm{m}$ membrane filter. RIF concentration was determined spectrophotometry at $\lambda_{\max }$ $479 \mathrm{~nm}$ using a UV-spectrophotometer (PG Instruments 
Ltd., Leicestershire, UK). The solvents studied were water, phosphate-buffered saline (PBS pH 7.4), methanol, ethanol, isopropyl alcohol, and propylene glycol. None of these solvents interfered with drug assay at the wavelength used. The experiments were performed in triplicate.

\section{Preparation of RIF NS}

RIF NS was prepared through the antisolvent nanoprecipitation technique previously reported by Zhang et al. ${ }^{22}$ In brief, RIF saturated organic solution was added to deionized water, as the nonsolvent phase, containing a suitable stabilizer at a ratio of 1:10. During the addition of the solvent phase, the aqueous phase was rapidly stirred at room temperature $\left(25 \pm 0.5^{\circ} \mathrm{C}\right)$ for 30 minutes.

\section{Screening of the process variables of nanoprecipitation technique}

In our preliminary studies, the process variables, for example, the type of solvent, stabilizer, stirrer type, and solvent flow rate of the nanoprecipitation technique, were investigated. The determining factors for optimization were the NS particle size along with its polydispersity index (PdI).

\section{Selection of stabilizer type}

The ability of different stabilizers to produce and stabilize RIF NS was utilized as a screening parameter to select the most suitable stabilizer. RIF NS was prepared using methanol as an organic solvent. Two classes of stabilizers were evaluated, namely, surfactants and polymeric stabilizers. The surfactants used were $0.5 \% \mathrm{w} / \mathrm{v}$ Poloxamer ${ }^{\circledR} 188$ and 407 , Tween ${ }^{\circledR} 80$, $\mathrm{SLS}^{24,25}$ and $1 \% \mathrm{w} / \mathrm{v}$ lecithin ${ }^{26}$ while $0.5 \% \mathrm{w} / \mathrm{v}$ HPMC $15 \mathrm{cp},{ }^{27} 0.2 \% \mathrm{w} / \mathrm{v}$ of HPMC $4,000 \mathrm{cp},{ }^{28} \mathrm{PVA},{ }^{19}$ and $0.4 \% \mathrm{w} / \mathrm{v}$ polyvinly pyrrolidone ${ }^{29}$ were the polymers chosen (Table 1 ).

\section{Selection of the stirrer type}

In order to study the effect of various stirring methods on RIF NS particle size, $0.2 \% \mathrm{w} / \mathrm{v}$ PVA as a stabilizer and methanolic solution were used to prepare RIF NS. Four stirring methods, specifically, magnetic stirring (RH basic IKA Labortechnik, Staufen, Germany), homogenization with rotor and stator homogenizer (T25 digital ULTRA-TURRAX ${ }^{\circledR}$, Staufen, Germany), mechanical stirring with an overhead stirrer with a blade of $5 \mathrm{~cm}$ length (RW 47 digital, IKA, Staufen, Germany), and ultrasound waves from a sonicator bath (Julabo Sonicator, model USR-3, Seelbach, Germany), were evaluated. NSs were stirred at room temperature $\left(25 \pm 0.5^{\circ} \mathrm{C}\right)$ for 30 minutes.

\section{Selection of the solvent flow rate}

RIF NS was prepared utilizing a sonicator bath for 30 minutes where RIF methanolic solution was added to the antisolvent aqueous solution containing $0.2 \% \mathrm{w} / \mathrm{v}$ PVA at three different flow rates (Table 2). The whole organic RIF solution was poured at once, the organic RIF solution was added at a flow rate of $2 \mathrm{~mL} / \mathrm{min}$ into the antisolvent solution, or both solvent and antisolvent solutions were simultaneously injected using syringes at the rate of $0.5 \mathrm{~mL} / \mathrm{min}$.

\section{Optimization of formulation parameters of RIF NS}

With the intention of studying and optimizing some formulation parameters, specifically, RIF methanolic solvent:antisolvent volume ratio (S:AS), concentration of PVA, and sonication time, eight RIF NS formulae were prepared by antisolvent nanoprecipitation method (Table 3 ). The effect of each parameter on the NS characteristics was independently studied. The RIF methanolic S:AS ratio was varied between 1:5-1:15, PVA concentration was either $0.1 \%, 0.2 \%, 0.4 \%$, or $0.6 \% \mathrm{w} / \mathrm{v}$, and sonication time was 5,15 , and 30 minutes.

\section{Physicochemical characterization of RIF NS Morphological study of the NS}

Particle morphological characteristics of the RIF NS $\left(\mathrm{F}_{5}\right)$ was observed using TEM (JEM-100 CX, JEOL, Tokyo, Japan) at

Table I Effect of stabilizers on the particle size and PdI of RIF NS, mean \pm SD ( $n=3)$

\begin{tabular}{lllll}
\hline Stabilizer type & Stabilizer & Concentration $(\%$ w/v) & Particle size (d.nm) & Pdl \\
\hline Polymer & PVA & 0.2 & $262.4 \pm 2.475$ & $0.326 \pm 0.009$ \\
& PVP K30 & 0.4 & $310.0 \pm 6.08 \mathrm{I}$ & $0.437 \pm 0.063$ \\
& HPMC (4,000 CP) & 0.2 & $473.5 \pm 2.192$ & $0.610 \pm 0.079$ \\
Surfactant & HPMC (I5 cP) & 0.5 & $553.3 \pm 7.637$ & $0.492 \pm 0.047$ \\
& Poloxamer 407 & 0.5 & $359.0 \pm 1.340$ & $0.473 \pm 0.093$ \\
& Lecithin & 1.0 & $413.0 \pm 1.273$ & $0.627 \pm 0.021$ \\
& SLS & 0.5 & $415.5 \pm 2.121$ & $0.596 \pm 0.006$ \\
& Poloxamer I88 & 0.5 & $495.0 \pm 1.320$ & $0.669 \pm 0.084$ \\
& Tween 80 & 0.5 & $842.0 \pm 1.313$ & $0.703 \pm 0.01 \mathrm{I}$ \\
\hline
\end{tabular}

Abbreviations: Pdl, polydispersity index; RIF NS, rifampicin nanosuspension; SD, standard deviation; PVA, polyvinyl alcohol; PVP K30, polyvinyl Pyrrolidone k30; HPMC, hydroxy propyl methylcellulose; SLS, sodium lauryl sulfate. 
Table 2 Effect of the stirring devices and solvent flow rates on the size and polydispersity index (PdI) of RIF NS, mean \pm SD ( $n=3$ )

\begin{tabular}{|c|c|c|c|c|c|c|c|}
\hline & \multicolumn{4}{|l|}{ Stirring devices } & \multicolumn{3}{|c|}{ Solvent flow rate } \\
\hline & Overhead stirrer & Magnetic stirrer & Homogenizer & Sonicator bath & $0.5 \mathrm{~mL} / \mathrm{min}$ & $2 \mathrm{~mL} / \mathrm{min}$ & On spot \\
\hline Particle size (d.nm) & $353 \pm 4.24$ & $262 \pm 2.48$ & $236 \pm 1.48$ & $173 \pm 3.65$ & $327 \pm 4.53$ & $221 \pm 4.38$ & $173 \pm 3.65$ \\
\hline Pdl & $0.436 \pm 0.07$ & $0.326 \pm 0.01$ & $0.456 \pm 0.01$ & $0.21 \pm 0.01$ & $0.502 \pm 0.01$ & $0.442 \pm 0.01$ & $0.2 \mathrm{I} \pm 0.04$ \\
\hline
\end{tabular}

Abbreviations: RIF NS, rifampicin nanosuspension; SD, standard deviation.

$80 \mathrm{kV}$. One drop of the NS was placed on a copper-coated grid leaving a thin film. Prior to TEM examination, the grid was left to dry at room temperature.

\section{Particle size and size distribution analysis}

The z-average diameter and size distribution in terms of PdI of the freshly prepared RIF NS were measured using a dynamic light scattering technique of a NanoZS/ZEN3600 Zetasizer (Malvern Instruments Ltd., Malvern, UK) equipped with Malvern PCS software (version 6.2). Each measurement was carried out in triplicate.

\section{ZP measurements}

The NS was evaluated by zeta potential (ZP) measurements using Malvern Zetasizer (Malvern Instruments Ltd.). Each sample was analyzed after 10-fold dilution with deionized water. Each diluted sample was added to the capillary cell equipped with platinum electrodes. The electrophoretic mobility of the particles was measured and converted into ZP value using the Dispersion Technology Software built into the Malvern zetasizer (version 6.2).

\section{Solubility determination}

Improvement of RIF saturated solubility is one of the essential merits of NS compared to other traditional formulations. Excess amount of free RIF, RIF NS $\left(\mathrm{F}_{5}\right)$, and the physical mixture of RIF and PVA was added to amber glass vials containing $5 \mathrm{~mL}$ of deionized water. The vials were shaken in a thermostatically controlled water bath (GFL, type 1083, GFL Gesellschaft für Labortechnik mbH, Burgwedel, Germany) at $37 \pm 0.5^{\circ} \mathrm{C}$ at $100 \mathrm{rpm}$. After equilibrium achievement (24 hours), the collected samples were centrifuged at $12,000 \mathrm{rpm}$ for 1 hour at $4{ }^{\circ} \mathrm{C}$ (Sigma Laboratory Refrigerated Centrifuge, Model 3K-30, Osterode am Harz, Germany). Then the supernatant was removed and filtrated through a $0.22 \mu \mathrm{m}$ syringe filter. The drug content in the samples was assayed using UV-spectrophotometer (PG Instruments Ltd.) at the predetermined $\lambda_{\max }$.

\section{In vitro dissolution studies}

In vitro dissolution behavior of RIF from the NS was assessed using the USP XXIV dissolution apparatus II, Type PTWS3 (Pharma Test, Hainburg, Germany). The RIF NS (equivalent to $50 \mathrm{mg}$ drug) was sprinkled on the surface of $300 \mathrm{~mL}$ PBS (pH 7.4) as the dissolution medium. ${ }^{15}$ The bath temperature and paddle stirring rate were set at $37 \pm 0.5^{\circ} \mathrm{C}$ and $100 \mathrm{rpm}$, respectively. At predetermined time intervals, $5 \mathrm{~mL}$ of the dissolution medium was withdrawn and compensated by an equal volume of fresh warm dissolution medium. Each sample was filtered through a $0.22 \mu \mathrm{m}$ syringe filter and analyzed for its RIF content spectrophotometrically at $\lambda_{\text {max }}$ $474 \mathrm{~nm}$. All runs were performed in triplicate.

Table 3 Optimization of formulation parameters of RIF NS, mean \pm SD $(n=3)$

\begin{tabular}{|c|c|c|c|c|}
\hline Formula & Factors & Particle size (d.nm) & PdI & Zeta potential $(\mathrm{mV})$ \\
\hline & S:AS ratio ${ }^{a}$ & & & \\
\hline$F_{1}$ & $\mathrm{I}: 5$ & $210.4 \pm 1.556$ & $0.365 \pm 0.038$ & $-14.1 \pm 0.029$ \\
\hline $\mathrm{F}_{2}$ & $1: 10$ & $|73.5 \pm 5.16|$ & $0.327 \pm 0.004$ & $-15.6 \pm 0.018$ \\
\hline \multirow[t]{2}{*}{$\mathrm{F}_{3}$} & $1: 15$ & $115.7 \pm 0.954$ & $0.325 \pm 0.04 I$ & $-18.3 \pm 0.022$ \\
\hline & PVA conce & & & \\
\hline $\mathrm{F}_{4}$ & $0.1 \% \mathrm{w} / \mathrm{v}$ & $|63.2 \pm 0.35|$ & $0.184 \pm 0.015$ & $-14.4 \pm 0.072$ \\
\hline $\mathrm{F}_{3}$ & $0.2 \% \mathrm{w} / \mathrm{v}$ & $115.7 \pm 0.954$ & $0.327 \pm 0.04 I$ & $-18.3 \pm 0.022$ \\
\hline $\mathrm{F}_{5}$ & $0.4 \% \mathrm{w} / \mathrm{v}$ & $101.7 \pm 0.208$ & $0.523 \pm 0.001$ & $-26.1 \pm 0.024$ \\
\hline \multirow[t]{2}{*}{$\mathrm{F}_{6}$} & $0.6 \% \mathrm{w} / \mathrm{v}$ & $204.0 \pm 13.70$ & $0.659 \pm 0.055$ & $-20.2 \pm 0.030$ \\
\hline & Sonication & & & \\
\hline $\mathrm{F}_{7}$ & 5 minutes & $256.3 \pm 15.98$ & $0.398 \pm 0.074$ & $-12.8 \pm 0.039$ \\
\hline $\mathrm{F}_{8}$ & 15 minutes & $179.4 \pm 1.747$ & $0.278 \pm 0.023$ & $-17.5 \pm 0.095$ \\
\hline $\mathrm{F}_{5}$ & 30 minutes & $101.7 \pm 0.208$ & $0.523 \pm 0.001$ & $-26.1 \pm 0.024$ \\
\hline
\end{tabular}

Notes: ${ }^{\mathrm{a}} \mathrm{F}_{1}-\mathrm{F}_{3}$ were prepared using $0.2 \% \mathrm{w} / \mathrm{v}$ PVA and 30 -minute sonication time. ${ }^{\mathrm{b}} \mathrm{F}_{4}-\mathrm{F}_{6}$ were prepared using I:I5 S:AS ratio and 30 -minute sonication time. ${ }^{\mathrm{C}} \mathrm{F}_{7}$ and $\mathrm{F}_{8}$ were prepared using I:I5 S:AS ratio and 0.4\% w/v PVA.

Abbreviations: RIF NS, rifampicin nanosuspension; SD, standard deviation; Pdl, polydispersity index; PVA, polyvinyl alcohol; S:AS, solvent:antisolvent volume ratio. 


\section{RIF-polymer compatibility studies}

In order to assess the physical state of RIF and detect any possible interaction between the NS components, the following characterizations were investigated.

\section{Differential scanning calorimetry}

Thermograms of free RIF, PVA, their physical mixture, and the RIF NS $\left(\mathrm{F}_{5}\right)$ were recorded using DSC 6 differential scanning calorimeter (PerkinElmer Inc., Waltham, MA USA). A sample of $5 \mathrm{mg}$ was weighed into a standard aluminum pan and heated at a rate of $10^{\circ} \mathrm{C} / \mathrm{min}$ over a temperature range of $25^{\circ} \mathrm{C}-300^{\circ} \mathrm{C}$. The instrument was calibrated with indium and dry nitrogen was used as a carrier gas with a flow rate of $40 \mathrm{~mL} / \mathrm{min}$. The main transition temperature was recorded and the enthalpy values were automatically calculated.

\section{Fourier transform infrared spectroscopy}

Fourier transform infrared spectroscopy spectra of free RIF, PVA, their physical mixture, and the RIF NS $\left(\mathrm{F}_{5}\right)$ were obtained using PerkinElmer Fourier transform infrared spectroscopy (PerkinElmer Inc., Waltham, MA USA). Each sample was mixed with dry infrared (IR) grade of crystalline potassium bromide then compressed at 10 tons in a hydraulic press to form a thin disk. Each sample was scanned within the spectral region of 4,000 and $400 \mathrm{~cm}^{-1}$ with a resolution of $4 \mathrm{~cm}^{-1}$.

\section{Physical stability of NS}

A short-term storage was performed to assess the physical stability of the RIF NS $\left(\mathrm{F}_{5}\right)$. RIF NS was stored in capped amber glass vials at two different temperatures $\left(4^{\circ} \mathrm{C}\right.$ and $25^{\circ} \mathrm{C}$ representing the refrigerator and room temperature, respectively) for a period of 3 months. The particle size, $\mathrm{PdI}$, and ZP of the samples were monitored on the day of preparation and followed on each month.

\section{In vitro cytotoxicity study}

To ensure the safety of the drug delivery system, cytotoxicity of the RIF NS $\left(\mathrm{F}_{5}\right)$ as well as free RIF was assessed using the 3(4,5-dimethylthiazol-2-yl)-2,5-diphenyltetrazolium bromide (MTT) assay on lung epithelial cells. The human alveolar basal epithelial cells (A549 cells) were cultured in F-12 Ham supplemented with $10 \%$ fetal bovine serum, $1 \%$ glutamine, and $100 \mu \mathrm{g} / \mathrm{mL}$ penicillin/streptomycin antibiotics. The cells were maintained in a humidified air atmosphere $\left(37^{\circ} \mathrm{C}, 95 \%\right.$ humidity, and $5 \% \mathrm{CO}_{2}$ ) as previously reported. ${ }^{30}$

The A549 cells were seeded into a 96-well plate at a density of $1 \times 10^{4}$ cells/well for 24 hours. Then, the cells were treated for another 24 hours with various concentrations of the free drug or the selected RIF NS. The cell viability after treatment was examined by the MTT assay. Briefly, MTT labeling reagent $(0.5 \mathrm{mg} / \mathrm{mL})$ was added to each well and incubated at $37^{\circ} \mathrm{C}$ for further 4 hours. Subsequently, the formed formazan crystals were quantified by spectrophotometry at $570 \mathrm{~nm}$ with a microplate reader (Synergy 4, ReaderBioTek Instruments, Bernareggio, Italy). The percentage of cell viability related to control cells incubated with culture medium only was determined by the following equation:

$$
\% \text { cell viability }=\frac{\mathrm{A}_{\text {(test) }}}{\mathrm{A}_{\text {(control) }}} \times 100
$$

where $\mathrm{A}_{\text {(test) }}$ is the absorbance obtained from the test sample and $\mathrm{A}_{\text {(control) }}$ is the absorbance obtained from untreated cells. The MTT assay for each concentration was performed in triplicate.

\section{Results and discussion Process variables of the nanoprecipitation technique}

\section{Selection of RIF solvent}

Screening of RIF solvent is crucial for the development of nanocrystals. During the nanoprecipitation process, the solvent that achieves the highest RIF solubility, will resulted in a higher drug concentration which leads to greater degree of supersaturation creating a high nucleation rate thereby forming smaller crystals. ${ }^{31}$ In the current work, six different solvents, namely, water, PBS pH 7.4, propylene glycol, isopropanol, ethanol, and methanol, were compared according to their power to solubilize RIF. The highest solubilization capacity was attained with methanol $(27 \pm 4.0415 \mathrm{mg} / \mathrm{mL})$, followed by ethanol $(18.8 \pm 3.0115 \mathrm{mg} / \mathrm{mL})$. Otherwise, water and PBS pH 7.4 showed the lowest solubilizing power $(0.106 \pm 0.0283$ and $1.87 \pm 0.3404 \mathrm{mg} / \mathrm{mL}$, respectively) as illustrated in Figure 1.

Methanol exhibited a greater capacity for dissolving both polar and nonpolar compounds with less toxicity and being easily removed as it is volatile, preventing instability of nanocrystal formulations. Several reports showed that Ostwald ripening and particle growth were the consequences of residual organic solvents. ${ }^{32,33}$ Therefore, methanol was chosen as a RIF suitable solvent for NS preparation.

\section{Selection of the stabilizer}

During the formulation of NS, the stabilizer presence is an essential component whatever the technique followed. 


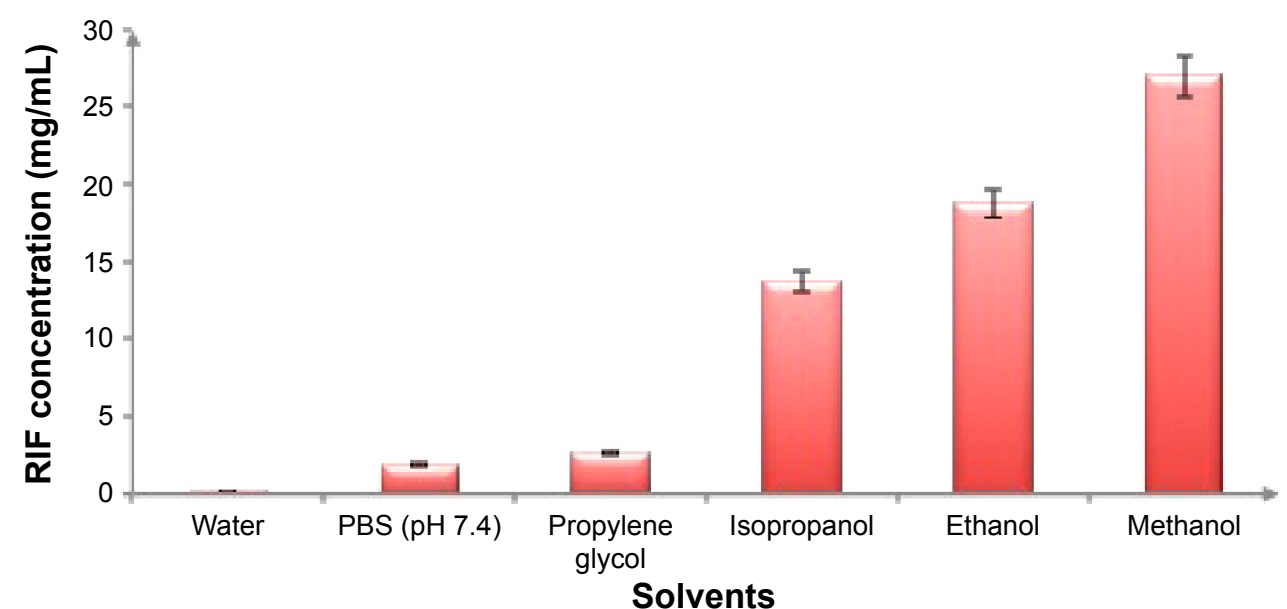

Figure I Solubility of RIF $(\mathrm{mg} / \mathrm{mL})$ in various solvents at $37 \pm 0.5^{\circ} \mathrm{C}$, mean $\pm \mathrm{SD}(\mathrm{n}=3)$.

Abbreviations: RIF, rifampicin; PBS, phosphate-buffered saline; SD, standard deviation.

The stabilizer should interact with the drug particle surface efficiently and prevent Ostwald's ripening and agglomeration of the NS particles with the aim of forming a physically stable NS formulation by providing either steric or ionic barriers. ${ }^{34}$ The most popular stabilizers used are surfactants, either ionic (SLS) or nonionic (poloxamer 188, poloxamer 407, and Tween 80). Moreover, lecithin and cholic acid derivatives as naturally occurring surfactants are frequently applied. Examples of polymeric stabilizers used are HPMC, acacia gum, hydroxy propylcellulose, PVA, and polyvinyl pyrrolidone. Electrostatic stabilization is a mechanism in which ionic surfactants are attributed to stabilize drug nanocrystals. In contrast, nonionic surfactants and polymers have been reported to facilitate the stability of NS via steric mechanism which depends on both the length and MW of steric stabilizer used. ${ }^{34}$ Nevertheless, recent research dealing with stability of NS has proved that the stabilization extent produced by polymers is different from that produced by surfactants. Polymeric stabilizers are adsorbed on the particle forming a thick layer with full surface coverage thus preventing or slowing drug molecule attachments to the dispersed particle. This adsorption may reduce the formation of large crystals from smaller ones (Ostwald's ripening). ${ }^{35}$ Additionally, it was found that the adsorbed polymer did not affect the crystal structure of the drug particles, in contrast to small MW surfactants. ${ }^{15}$

Different polymeric and surfactant stabilizers were evaluated during this study, namely, SLS, soybean lecithin, poloxamers, Tween 80, HPMC, PVP K30, and PVA.

Besides the type of the stabilizer, its concentration plays an important role in the stabilization process. The amount of stabilizing agent should be sufficient to obtain a full coverage barrier surrounding the surface of nanocrystals. In the current work, the concentration of various stabilizers was selected according to their optimum reported concentrations in the literature.

One of the most significant characteristics for successful stabilizer use during NS formation is the development of a small particle size of the drug crystals. ${ }^{16}$ The particle size and PdI of prepared RIF NS are considered to be crucial factors in determining the rate and extent of drug release, as well as the stability of the system. PdI is an indication of size distribution of the NS population and should be in the range of $0-0.2$ to ensure the uniformity and homogeneity of drug delivery system. ${ }^{36}$ It was found that the particle size of NS prepared without the addition of any stabilizer was $6 \mu \mathrm{m}$ as a consequence of particle aggregation. Conversely, as shown in Table 1, the studied stabilizers at their optimum concentrations were able to achieve nano-sized particles. The most effective particle size reduction was observed with the formulation containing $0.2 \% \mathrm{w} / \mathrm{v}$ PVA $(262.4 \mathrm{~nm})$ while the largest NS particle size together with the widest distribution (PdI, 0.7035) resulted upon the utilization of Tween 80 as a stabilizer $(842 \mathrm{~nm})$. This may be due to its small MW $(1,310 \mathrm{~g} / \mathrm{mol})$ which reflected on the formation of a thin adsorption layer around the RIF crystals that was not sufficient to stabilize the system leading to crystal aggregation and excessive large particle formation. ${ }^{37}$ On the contrary, it was noticed that lecithin and SLS were able to form NS with a nonsignificant difference in particle size $(\sim 414 \mathrm{~nm})$, as both surfactants prevented particle agglomeration via electrostatic repulsion. Morakul et $\mathrm{al}^{38}$ reported that SLS stabilized clarithromycin NS and reduced its particle size to $851 \mathrm{~nm}$ in comparison to other surfactants. Moreover, Gao et $\mathrm{al}^{26}$ 
achieved the formation of curcumin NS with the smallest particle size $(250 \mathrm{~nm})$ using lecithin as a stabilizer. However, both SLS and lecithin cannot be used solely to form homogeneous RIF NS as their PdI was large $(0.596 \pm 0.006$ and $0.627 \pm 0.021$, respectively), which indicated a broad indicates size distribution (Table 1).

Poloxamers inhibit crystal growth chiefly through the hydrophobic interaction of polypropylene oxide group in its polymeric structure. Comparing their two grades, Poloxamer 407 produced smaller nanocrystals in contrast to Poloxamer 188. This variation can be linked to their difference in MW. The higher MW of Poloxamer 407 (MW 14,600 g/mol) increased its ability to cover RIF particle surfaces more than that of Poloxamer 188 (MW 7,680 g/mol). These results were in accordance to those reported by Ghosh et al. ${ }^{39}$

From Table 1, HPMC (4,000 cp) of higher viscosity resulted in the formation of smaller particles $(473.5 \pm 2.192 \mathrm{~nm})$ but with a larger particle distribution index $(0.610 \pm 0.079)$ compared to that of the lower viscosity. HPMC polymers are able to induce steric stabilization of the NS through their absorption on the drug particle surface via their hydrophilic and hydrophobic components, namely, hydroxy propyl and methoxyl groups, respectively. ${ }^{27} \mathrm{~A}$ small NS particle size was obtained in the presence of $0.4 \% \mathrm{w} / \mathrm{v}$ PVP K30 (310.0 46.081 $\mathrm{nm})$ that indicates its ability to shield particle surface more efficiently than other cellulosic derivatives (Table 1). The observed results were contrary to that mentioned by Verma et $\mathrm{al}^{40}$ who related the steric hindrance capability to the geometrical differences between HPMC and polyvinyl pyrrolidone without considering the viscosity influence. Moreover, surface energy of both the drug and stabilizer measured by contact angle measurement plays an important role in the selection of a suitable and efficient NS stabilizer as reported by Choi et al. ${ }^{41}$ Hence, the formulation containing $0.2 \% \mathrm{w} / \mathrm{v}$ PVA was thereby used for further investigations having the smallest particle size $(262.4 \pm 2.475 \mathrm{~nm})$ with the lowest PdI (0.326 \pm 0.009$)$.

\section{Selection of the stirring method}

During antisolvent precipitation process, the solvent and antisolvent mixing is essential to control the particle size and distribution pattern of the precipitated drug crystals. ${ }^{18}$ Homogeneous mixing influences the degree of supersaturation, which impacts the nucleation and particle growth rate. The stirring device is one of the factors that affects mixing efficiency ${ }^{32}$ For this purpose, various mixing/stirring devices (namely, magnetic stirrer, rotor stator homogenizer, over-head stirrer, and sonicator bath) were compared depending on both the particle size and PdI of the RIF NS. As shown in Table 2, the four mixing devices achieved a particle size within a range of $173-353 \mathrm{~nm}$ with PdI ranging from 0.215 to 0.456 .

Sonication proved to produce the lowest nanosized particles $(173 \mathrm{~nm})$ with a narrow size distribution $(0.21)$, in contrast to the other stirrers. Figure 2 exhibited a unimodal size distribution of the nanocrystal bearing RIF prepared using the sonicator bath as the mixing device. Therefore, the formulation containing $0.2 \% \mathrm{w} / \mathrm{v}$ PVA as a stabilizer prepared with sonication-antisolvent nanoprecipitation method was further investigated. Ultrasound waves have been shown to be capable of producing a higher degree of supersaturation resulting in a higher nucleation rate and smaller precipitated particles sizes. Moreover, Luque de castro and Priego-Capote ${ }^{42}$ reported that these waves have the ability to create cavitation and acoustic streaming in the solution, thereby reducing the crystal's induction time, increasing the nucleation rate, and suppressing the metastable zone width. This cavitation also generates shock waves which preclude the bonding and contact between the crystals and, hence, prevent their agglomeration and reduce their size.

Zhang et $\mathrm{al}^{22}$ prepared successfully camptothecin nanocrystals with a sonication-precipitation method. The prepared nanocrystals show rod- or needle-like shape with a uniform particle size (200-700 nm) and were stable for 6 months at low temperature. In another study conducted by Xia et al, ${ }^{19}$ the hydrodynamic diameter of flaked-shaped nitrendipine NS prepared by ultrasonication-precipitation method was $209 \mathrm{~nm}$ with a $\mathrm{ZP}$ of $-14 \mathrm{mV}$.

\section{Screening study on the effect of solvent flow rate on NS properties}

It was reported that the rate of nucleation and crystal growth depend mainly on the solvent flow rate. ${ }^{18}$ Under these consequences, three different flow rates of RIF methanolic solutions were investigated. From the results computed in Table 2, it could be inferred that increasing of the flow rate was inversely proportional to both the particle size

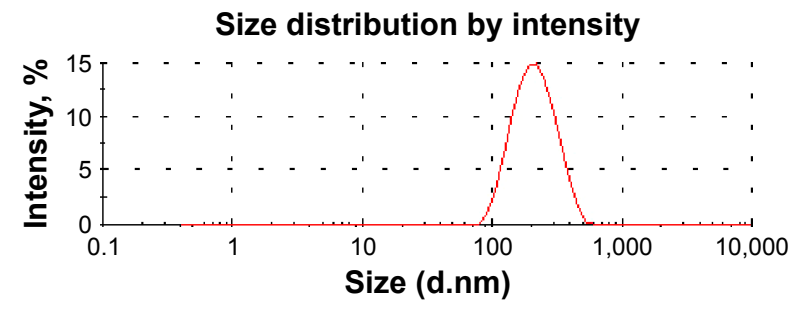

Figure 2 Particle size distribution by intensity of rifampicin nanosuspension prepared with ultrasonication-antisolvent precipitation method. 
and PdI. The NS size was decreased from 327 to $173 \mathrm{~nm}$ and the PdI was shifted from 0.502 to 0.21 by adding on spot the methanolic solution into antisolvent solution. At a high flow rate, both solvent and antisolvent solutions were mixed rapidly together, increasing their mixing extent per unit time and producing a rapid supersaturation and nucleation, thus resulting in the formation of smaller crystals. ${ }^{43}$ A similar finding was proved by Kakran et al, ${ }^{20}$ where the particle size of quercetin nanoparticles was decreased from 255 to $170 \mathrm{~nm}$ as the solvent flow rate was increased from 2 to $8 \mathrm{~mL} / \mathrm{min}$.

Therefore, after these preliminary studies, RIF NS was prepared by pouring at once RIF methanolic saturated solution to an aqueous solution containing $0.2 \% \mathrm{w} / \mathrm{v}$ PVA as a stabilizer at a ratio $1: 10$ using a sonicator bath for 30 minutes at room temperature $\left(25 \pm 0.5^{\circ} \mathrm{C}\right)$.

\section{Optimization of formulation parameters of RIF NS}

To ensure good NS performance, several properties should be adjusted, for example, particle size, PdI, and ZP values. ZP measurements reflect the surface properties of the RIF NS which estimate the physical stability of NS being formed. In order to obtain a long-term stable NS, an absolute value of the ZP of approximately $\pm 30 \mathrm{mV}$ for electrostatically and $\pm 20 \mathrm{mV}$ for sterically stabilized systems is desired. ${ }^{44}$ These ZP values serves as an indicator for the creation of a high-energy thick diffusion barrier against the aggregation or agglomeration of dispersed particles. ${ }^{44}$ For these reasons, eight different formulations were prepared to study the influence of various formulation parameters of RIF NS, namely $\mathrm{S}$ :AS, stabilizer concentration and sonication time on particle size, PdI, and ZP (Table 3).

\section{Effect of S:AS ratio}

The selection S:AS ratio was reported to be a critical step in the antisolvent precipitation method. ${ }^{18}$ As illustrated in Table 3, upon increasing the S:AS volume ratio from 1:5 to $1: 15$, the particle size of RIF NS was decreased from 210.4 to $115.7 \mathrm{~nm}$, respectively. When the ratio of S:AS was increased, the degree of supersaturation was increased, resulting in a greater nucleation rate leading to the formation of smaller crystals. At low S:AS volume ratio, the presence of a higher solvent phase induces the particle growth, which would increase the particle size. Furthermore, at this low ratio, the decreased amount of antisolvent may reduce the diffusion distance between the growing species; thus, the crystal growth will be dominant resulting in increased particle size. ${ }^{45}$ Therefore, the optimum S:AS volume ratio (1:15) was chosen for further investigations since it showed the smallest particle size, least PdI, and highest negative ZP value.

\section{Effect of the stabilizer concentration}

Realizing the crucial impact of the stabilizer on NS characteristics, the influence of its concentration on formulations particle size, PdI, and ZP was estimated (Table 3). Results demonstrated a significant reduction in particle size of RIF NS upon increasing PVA concentration from $0.1 \% \mathrm{w} / \mathrm{V}$ $\left(\mathrm{F}_{4}, 163 \mathrm{~nm}\right)$ to $0.4 \% \mathrm{w} / \mathrm{v}\left(\mathrm{F}_{5}, 101 \mathrm{~nm}\right)$. However, a further increase in PVA concentration showed an increase in the particle size of RIF NS ( $\left.\mathrm{F}_{6}, 204 \mathrm{~nm}\right)$. On the other hand, PdI values showed a significant increase from 0.184 to 0.659 as a result of elevation of PVA concentration. Moreover, the prepared nanocrystals were negatively charged with ZP values ranging from -14 to $-26 \mathrm{mV}$.

Results inferred that the use of $0.4 \% \mathrm{w} / \mathrm{v}$ PVA was decisive for full surface coverage to produce a stable crystal in the nanometric range. The lowest PVA concentration $\left(\mathrm{F}_{4}\right)$ may be insufficient for complete adsorption on the crystal whereas the use of the highest PVA concentration $\left(\mathrm{F}_{6}\right)$ increased the solution viscosity, which hinders the diffusion toward the interface of drug solvent and antisolvent solution. PVA being a nonionic polymer inhibits the crystal growth with the creation of a thick diffusive boundary layer around the nanocrystals that provides enough steric repulsion between the particles. ${ }^{19}$ Besides, the polymer molecules may be adsorbed on the crystal faces preventing their aggregation. ${ }^{34}$ The possible mechanism of polymer adsorption on the crystal surface may be by either formation of hydrogen bonds between the drug molecules and the polymer or the incorporation of drug molecules into crystal lattice. ${ }^{32}$ Thus, $0.4 \% \mathrm{w} / \mathrm{v}$ PVA was chosen as an optimum stabilizer concentration for further investigation.

\section{Effect of the ultrasonication time}

The influence of ultrasonication time on the performance of RIF NS $\left(\mathrm{F}_{5}, \mathrm{~F}_{7}\right.$, and $\left.\mathrm{F}_{8}\right)$ is depicted in Table 3. The prolongation of sonication time from 5 to 30 minutes resulted in a corresponding decrease in particle size from 256 to $101.7 \mathrm{~nm}$, respectively. A higher time of mixing between the solvent and the antisolvent solution may cause a rapid nucleation rate resulting in a reduction of particle size. ${ }^{46}$ Moreover, ZP measurements of the RIF NS showed a more negative value within the range -12.8 to $-26.1 \mathrm{mV}$ upon increasing the ultrasonication time, thus indicating a good colloidal stability. The ultrasonication time of 5 minutes was insufficient to 
produce a stable NS. Hence, the optimum ultrasonication time was 30 minutes.

\section{Physicochemical characterization of RIF NS}

\section{Morphological study of NS}

RIF NS containing $0.4 \% \mathrm{w} / \mathrm{v}$ PVA as a stabilizer $\left(\mathrm{F}_{5}\right)$ was assessed using TEM as illustrated in Figure 3. The photomicrograph of RIF NS revealed crystal-shaped particles that are uniformly distributed in the medium with a particle size of $100 \mathrm{~nm}$ or less. This particle size was in a good agreement with that obtained from the earlier particle size measurements (Table 3). Additionally, the micrograph exhibited nonaggregated stable crystals, which indicate a good coverage of RIF nanocrystal surface and subsequently inferred system stability. These results are in accordance with those obtained from ZP measurements (Table 3).

\section{RIF solubility determination}

The saturation solubility of free RIF, in comparison with its physical mixture with PVA and the RIF NS $\left(\mathrm{F}_{5}\right)$ in deionized water at $37 \pm 0.5^{\circ} \mathrm{C}$, is shown in Figure 4. Solubility value of the physical mixture of PVA and RIF exhibited a 25-fold increase in the RIF solubility due to the solubilization power of PVA. Moreover, the solubilization of RIF as nanocrystals showed a 50-fold greater than that of its powder and 25 -fold greater than the corresponding physical mixture. According to the above results, the NS formulations markedly improved the RIF apparent water solubility reaching to $472 \mathrm{mg} \%$. This significant enhancement in the drug solubility could be mainly ascribed to the progressive size reduction of the drug particle size with a corresponding increase in surface curvature and area. The result could be contended with the Ostwald-Freundlich equation, which revealed that

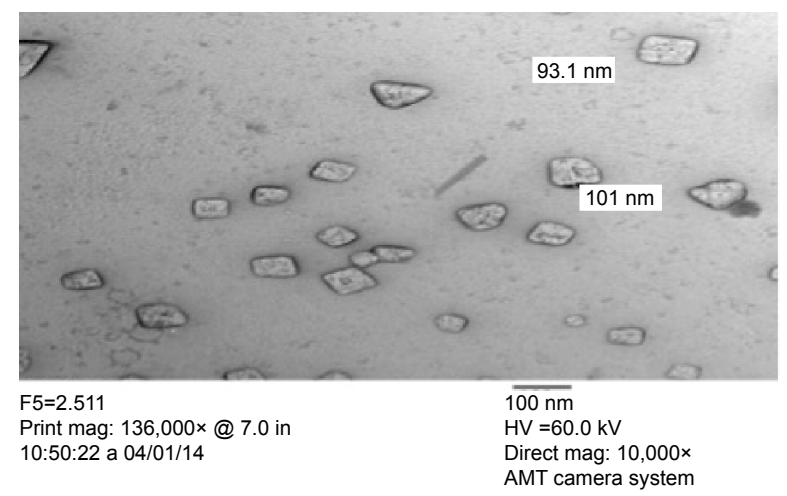

Figure 3 Transmission electron micrograph of the rifampicin nanosuspension $\left(\mathrm{F}_{5}\right)$ with magnification $10,000 \times$.

Abbreviations: mag, magnification; in, inch.

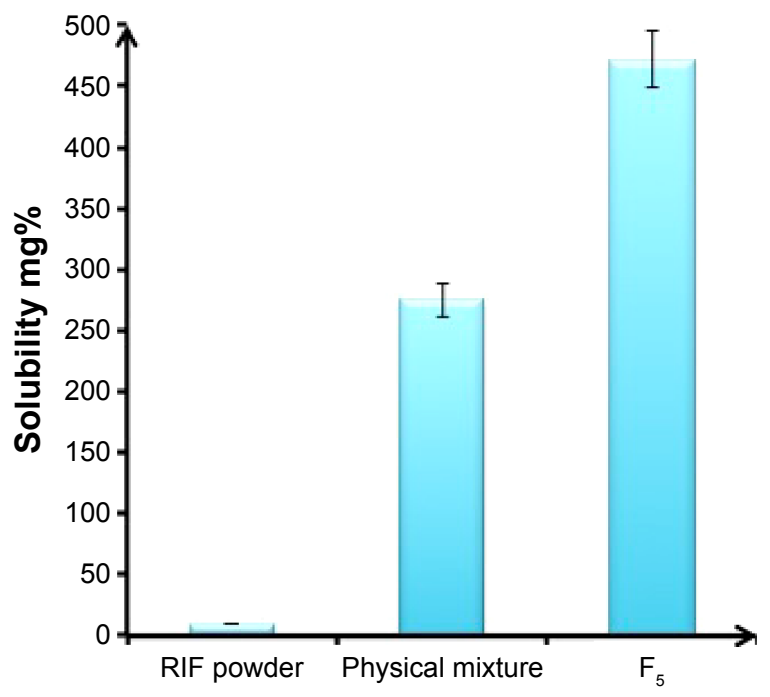

Figure 4 Saturation solubility of RIF powder, physical mixture and RIF NS $\left(\mathrm{F}_{5}\right)$ in deionized water at $37 \pm 0.5^{\circ} \mathrm{C}$, mean $\pm S D(n=3)$.

Abbreviations: RIF, rifampicin; RIF NS, rifapmicin nanosuspension; SD, standard deviation.

the improvement of drug solubility depends on its particle size. ${ }^{13}$ Hao et $\mathrm{al}^{21}$ reported that Amoitone $\mathrm{B}$ nanocrystals showed a three- and 15-fold increase in drug solubility in comparison to its physical mixture with Pluronic F68 and powder, respectively, due to the difference in particle size. As implied in Table 3, the particle size of the RIF nanocrystals $\left(\mathrm{F}_{5}\right)(101.7 \mathrm{~nm})$ was smaller than that of Amoitone B nanocrystals $(256.3 \mathrm{~nm})$. Thus, the improvement in drug solubility is clearly associated with reduction in drug particle size.

\section{In vitro dissolution studies}

One of remarkable properties of NS is the augmentation of drug saturation solubility and subsequently the increase in its dissolution rate. Hence, the in vitro release patterns of free RIF compared to that from different NSs prepared with variable drug/stabilizer ratios $\left(\mathrm{F}_{3}-\mathrm{F}_{5}\right)$ in PBS (pH 7.4) are depicted in Figure 5. The patterns exhibited a notable improvement in RIF dissolution from different NSs in contrast to the powdered drug. As illustrated in Figure 5, dissolution profiles of different RIF NS formulations $\left(\mathrm{F}_{3}-\mathrm{F}_{5}\right)$ showed a significant difference within the first 5 minutes. The results revealed that after 5 minutes, $70 \%, 85 \%$, and $93 \%$ of RIF was released from $\mathrm{F}_{4}\left(0.1 \%\right.$ PVA), $\mathrm{F}_{3}(0.2 \% \mathrm{PVA})$, and $\mathrm{F}_{5}(0.4 \%$ PVA), respectively. Such a difference in drug release profile could be mainly implied due to variations in particle size as shown in Table 3. Moreover, it was found that $79 \%, 90 \%$, and $97 \%$ of RIF was released from $\mathrm{F}_{4}, \mathrm{~F}_{3}$, and $\mathrm{F}_{5}$, respectively, within 10 minutes whereas $37 \%$ was only released from free RIF. Following Noyes-Whitney equation, further significant dissolution rate enhancement 


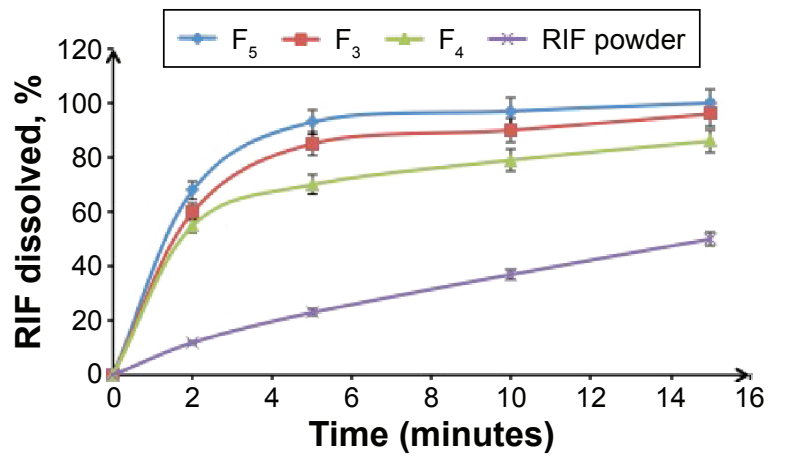

Figure 5 Dissolution profiles of RIF NS containing variable PVA concentrations $\left(\mathrm{F}_{3}-\mathrm{F}_{5}\right)$ in $\mathrm{PBS}(\mathrm{pH} 7.4)$ at $37 \pm 0.5^{\circ} \mathrm{C}$ compared to RIF powder, mean \pm SD $(n=3)$. Abbreviations: RIF, rifampicin; RIF NS, rifapmicin nanosuspension; PVA, polyvinyl alcohol; PBS, phosphate-buffered saline; SD, standard deviation.

of different NS could be attributed to particle size reduction with corresponding increase in surface area and improvement in saturated solubility. ${ }^{47}$ Furthermore, the nanometric size of drug particles may decrease the thickness of the diffusion layer between the surface of particle and bulk solution leading to an increase in the dissolution velocity, which could be explained by the Prandtl equation. ${ }^{21}$ This was in a good agreement with the previous results that confirm the choice of RIF NS $\left(\mathrm{F}_{5}\right)$ containing $0.4 \% \mathrm{w} / \mathrm{v}$ PVA as an optimum NS formulation for further characterization.

In comparison to the study of Son and McConville, ${ }^{10}$ where RIF dissolution rate was enhanced by polymorphic transformation into its dihydrated form and its release profile was 74\% within the first hour, the prepared RIF NS formulations showed a promising in-vitro dissolution enhancement.

\section{RIF-polymer compatibility studies Differential scanning calorimetry}

DSC analysis has been carried out to investigate any physical change in the crystalline state and thermal behavior of RIF upon the nanosizing process. The crystallinity state assessment will predict the physicochemical properties of the NS system as saturation solubility, dissolution, and physical stability. DSC thermograms of free RIF, PVA, their physical mixture, and the RIF NS $\left(\mathrm{F}_{5}\right)$ are illustrated in Figure 6. RIF has been postulated to exist in two different polymorphic states, Form I and Form II, which differ in their amorphous content. Agrawal et $\mathrm{al}^{48}$ found that Form I is more thermodynamically stable than Form II. As shown in Figure 6, the unprocessed RIF decomposes directly with a sharp exothermic peak at $263^{\circ} \mathrm{C}$ corresponding to the peak pattern of Form I. The DSC graph of PVA exhibited a broad endothermic peak at $172^{\circ} \mathrm{C}-199^{\circ} \mathrm{C}$ indicating its melting point.
DSC characteristic peaks of both free RIF and PVA were revealed in the thermogram of their physical mixture. The thermogram of the RIF NS revealed two endothermic and one exothermic peaks. The first endothermic peak at $\left(37^{\circ} \mathrm{C}-85^{\circ} \mathrm{C}\right)$ corresponds to the evaporation of physically absorbed water as reported by Son and McConville ${ }^{11}$ whereas the second one indicates the melting point of PVA. For RIF NS $\left(\mathrm{F}_{5}\right)$, RIF exothermic peak was broadened and shifted to a lower temperature of $240^{\circ} \mathrm{C}$ compared to that of free RIF. This phenomenon can be probably attributed to the partial transformation of the crystalline state to the amorphous form of RIF. Similarly, this finding was reported in another study where the peak of myricetin was shifted to a lower temperature when it was formulated as an NS. ${ }^{49}$ Moreover, both RIF-PVA physical mixture $(\Delta \mathrm{H}=69.6 \mathrm{~J} / \mathrm{g})$ and $\operatorname{RIF~NS}(\Delta \mathrm{H}=12.8 \mathrm{~J} / \mathrm{g})$ showed a decline in the enthalpy value of RIF in contrast to that of free RIF $(\Delta H=104.7 \mathrm{~J} / \mathrm{g})$. This decline can be explained by the formation of less ordered crystals that release a lesser amount of energy than those of the crystalline ones. ${ }^{50}$

\section{Fourier transform infrared spectroscopy}

For further detection of any possible interaction between RIF and the stabilizer molecules in the solid state, IR spectra of RIF, PVA, their physical mixture, and the RIF NS $\left(\mathrm{F}_{5}\right)$ are illustrated in Figure 7. RIF showed absorption bands at 3,482, 2,878, $1,726,1,643$, and $1,566 \mathrm{~cm}^{-1}$ attributable to hydroxyl, N-methyl, acetyl carbonyl, furanone carbonyl, and amide carbonyl groups, respectively. IR spectrum of RIF was corresponding to that of Form I as proved previously by DSC results, in which RIF Form I exhibited a characteristic single peak at $1,726 \mathrm{~cm}^{-1}$ in addition to a sharp absorption band at $3,482 \mathrm{~cm}^{-1}$.

The stabilizer PVA molecules exhibited a large absorption band between 3,550 and 3,200 $\mathrm{cm}^{-1}$ due to stretching the hydroxyl group from the intermolecular and intermolecular hydrogen bonds. Vibrational band stands for stretching C-H from alkyl groups are observed at a region between 2,840 and $3,000 \mathrm{~cm}^{-1}$. Furthermore, PVA revealed a characteristic peak at $1,140 \mathrm{~cm}^{-1}$, which refers to stretching of the etheric oxygen group.

All characteristic absorption bands of RIF were observed in both their physical mixture and RIF NS $\left(\mathrm{F}_{5}\right)$. However, in the IR spectrum of RIF NS, the peaks were noticeably broader. This is possibly due to hydrogen bond formation between the drug and stabilizer molecules.

\section{Physical stability}

NSs are essentially thermodynamically unstable systems. Thus, monitoring the stability of the RIF NS $\left(\mathrm{F}_{5}\right)$ is a critical 

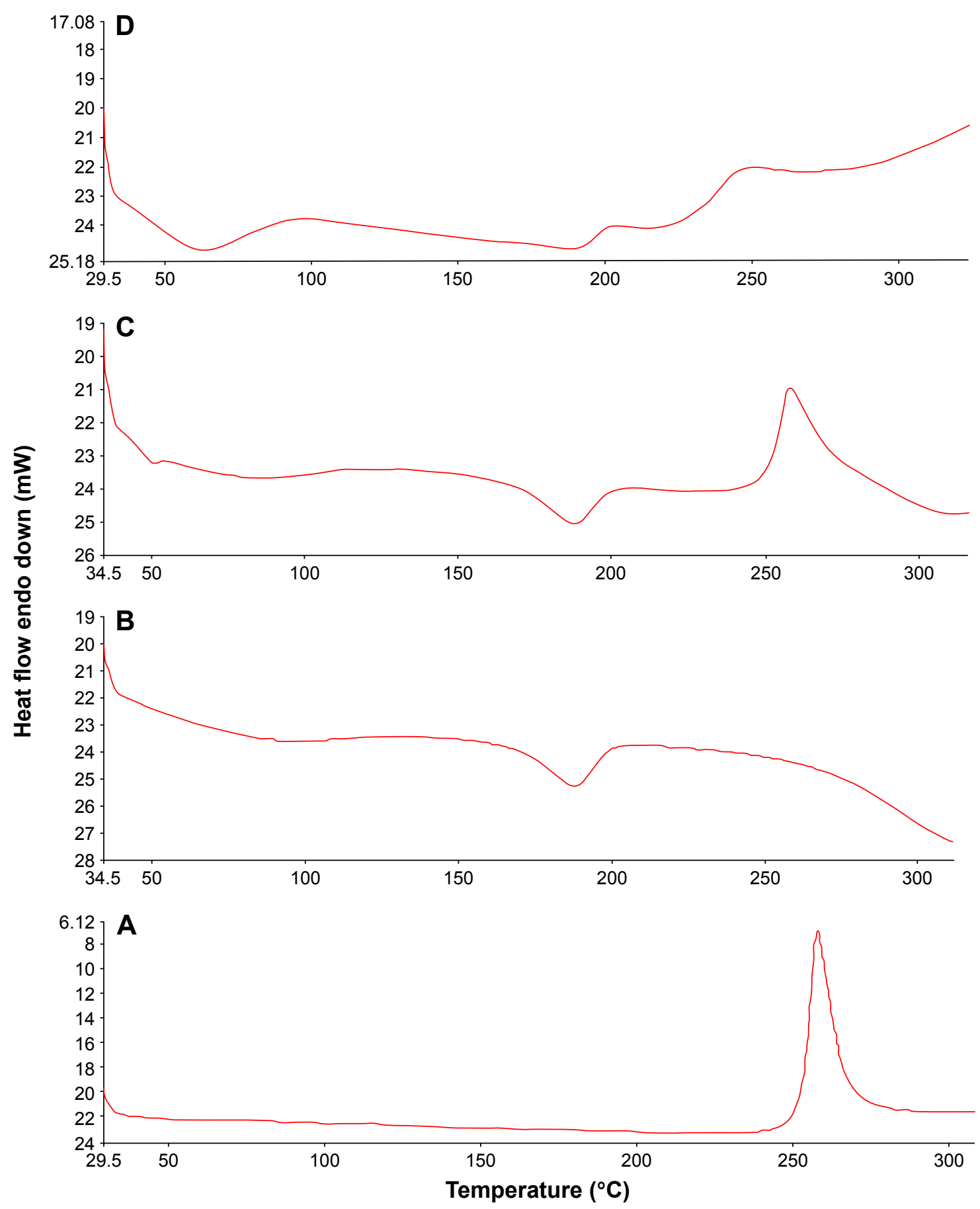

Figure 6 DSC thermograms of RIF (A), PVA (B), their physical mixture $(\mathbf{C})$, and RIF NS $\left(F_{5}\right)(\mathbf{D})$.

Abbreviations: DSC, differential scanning calorimetry; RIF, rifampicin; NS, nanosuspension; PVA, polyvinyl alcohol.

step. In order to predict the physical properties, physicochemical properties (particle size, PdI, and ZP) were assessed over a period of 3 months in two different storage temperatures as summarized in Table 4 . At low temperature $\left(4^{\circ} \mathrm{C}\right)$, the NS revealed no significant change in both particle size and PdI over a period of 3 months. On the contrary, storage at a higher temperature $\left(25^{\circ} \mathrm{C}\right)$ showed a gradual increase in particle size whereas the PdI revealed a slight decrease.
The increase in particle size could be attributed to Ostwald ripening in which small particles dissolved and redeposited on the surfaces of larger ones. At lower temperature, Ostwald ripening was retarded but at room temperature, this phenomenon was more facilitated where the redeposition of dissolved species to the larger particles became faster, increasing the particle size. Furthermore, after 3 months, the decrease in PdI suggested a unimodal particle size distribution but with 


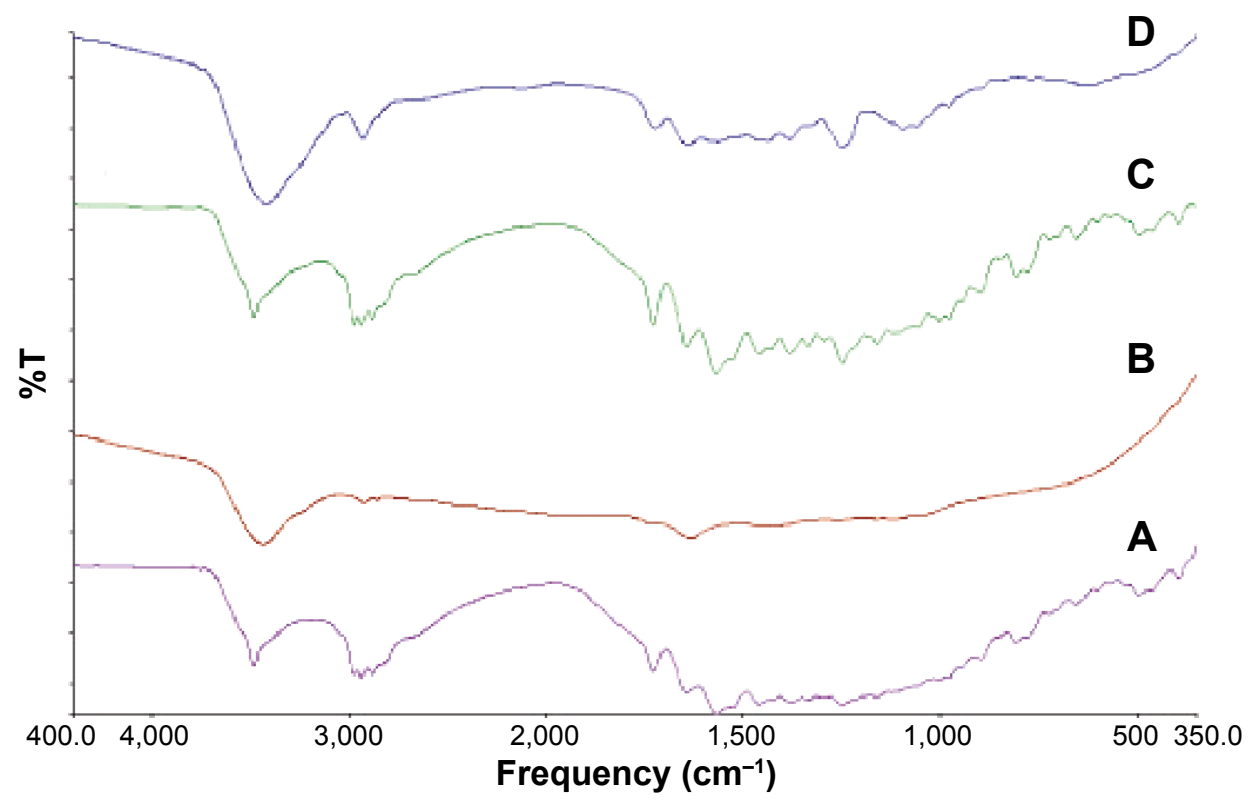

Figure 7 FTIR spectra of RIF (A), PVA (B), their physical mixture (C), and RIF NS $\left(F_{5}\right)$ (D).

Abbreviations: FTIR, Fourier transform infrared spectroscopy; RIF, rifampicin; NS, nanosuspension; PVA, polyvinyl alcohol; T, transmittance.

particles of larger size as depicted in Figure 8. Zhang et al ${ }^{22}$ observed similar results.

Additionally, the absolute value of ZP of nanocrystals became less negative over the period of storage time at $25^{\circ} \mathrm{C}$ while it shows a slight increase from -26 to $-20 \mathrm{mV}$ when stored at $4^{\circ} \mathrm{C}$. It was reported that the steric stabilization, provided by PVA, was more sensitive to the elevation of temperature. Thus, the aggregation of small crystals was mainly enhanced at ambient temperature. ${ }^{12}$ Hence, the storage temperatures clearly affected the physicochemical properties of RIF NS. The storage of RIF NS at low temperature $\left(4^{\circ} \mathrm{C}\right)$ proved to be superior in terms of physical stability relative to room temperature within the period of 3 months.

\section{In vitro cytotoxicity study}

NSs as drug delivery systems have attracted unique functional characteristics defined by their size-related properties. These outstanding features can affect their safety on tissues.
Therefore, their toxicity against A549 human alveolar epithelial cells has been investigated using in vitro MTT cytotoxicity assay. This assay had been known to indicate the level of mitochondrial dehydrogenase enzymes that is still metabolically active. The cell viability exhibited a concentration-dependent activity that decreased with raising RIF concentration. However, it was obvious that the percentage of cell cytotoxicity in the presence of RIF NS $\left(\mathrm{F}_{5}\right)$ was clearly lower than that obtained with free RIF at all the concentrations tested (Figure 9), which suggested the safety of the used excipients. The half maximal inhibitory concentration was obtained from the concentration-dependent cell viability curves. The half maximal inhibitory concentration values were 0.5 and $0.8 \mathrm{mg} / \mathrm{mL}$ for free RIF and RIF NS, respectively. These results could be attributed to a slower cellular uptake of nanocrystals in comparison to free RIF. The cellular uptake of nanocrystals may occur by either receptor-mediated or nonspecific endocytosis. ${ }^{51}$ The former, likely the clathrin- and caveolae-mediated endocytosis,

Table 4 Short-term physical stability study of RIF NS $\left(F_{5}\right)$ at two storage temperatures over 3 months

\begin{tabular}{|c|c|c|c|c|c|c|}
\hline \multirow[t]{2}{*}{ Time (month) } & \multicolumn{3}{|c|}{ Room temperature $\left(25 \pm 0.5^{\circ} \mathrm{C}\right)$} & \multicolumn{3}{|c|}{ Refrigerator $\left(4 \pm 0.5^{\circ} \mathrm{C}\right)$} \\
\hline & Particle size $(\mathrm{nm})$ & Zeta potential $(\mathrm{mV})$ & PdI & Particle size $(\mathrm{nm})$ & Zeta potential $(\mathrm{mV})$ & Pdl \\
\hline 0 & $101.7 \pm 0.208$ & $-26.1 \pm 0.024$ & $0.523 \pm 0.001$ & $101.7 \pm 0.208$ & $-26.1 \pm 0.024$ & $0.523 \pm 0.001$ \\
\hline Ist & $139.2 \pm 1.560$ & $-24.5 \pm 0.047$ & $0.515 \pm 0.014$ & $131.7 \pm 3.536$ & $-26.3 \pm 0.023$ & $0.543 \pm 0.016$ \\
\hline 2nd & $179.9 \pm 5.859$ & $-|8.1 \pm 0.05|$ & $0.455 \pm 0.024$ & $160.1 \pm 1.900$ & $-20.1 \pm 0.069$ & $0.546 \pm 0.056$ \\
\hline $3 r d$ & $198.8 \pm 8.556$ & $-17.9 \pm 0.070$ & $0.368 \pm 0.006$ & $166.5 \pm 7.436$ & $-20.2 \pm 0.089$ & $0.540 \pm 0.095$ \\
\hline
\end{tabular}

Note: Data presented as mean $\pm S D(n=3)$.

Abbreviations: RIF NS, rifampicin nanosuspension; SD, standard deviation; Pdl, polydispersity index. 

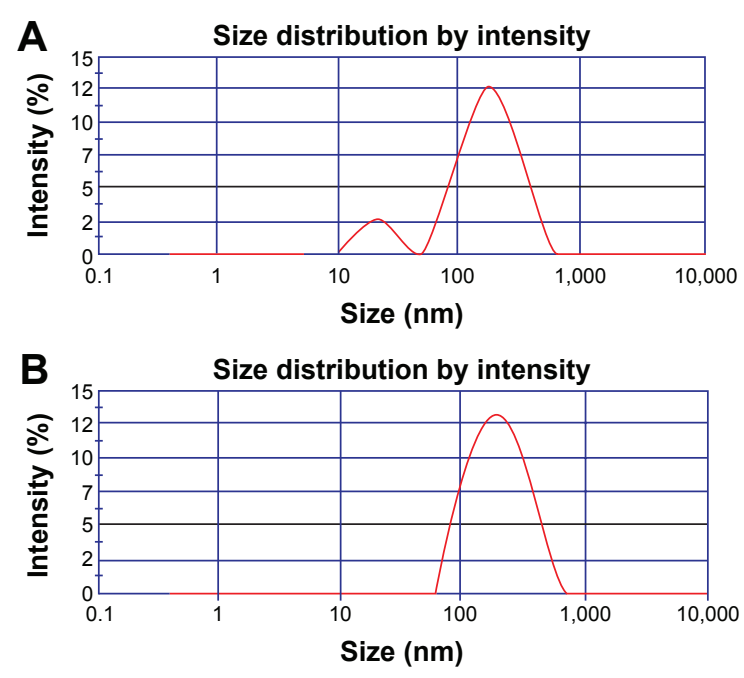

Figure 8 Particle size distribution by intensity of rifampicin nanosuspension $\left(F_{5}\right):(A)$ on the day of preparation and (B) after 3 months storage at $25 \pm 0.5^{\circ} \mathrm{C}$.

required a specific transport ligand while the latter depends on the morphological and physicochemical characteristics of nanocrystals. ${ }^{22,51}$ The surface charge of the nanocrystals implies an important role in the cellular uptake. Mahmoud et $\mathrm{al}^{52}$ proved that the cellular uptake of positively charged cellulose nanocrystals via human embryonic kidney 293 was superior to that of the negatively charged one. The authors attributed this to the repulsive force between the negatively charged cell membrane and nanocrystals. In accordance, the slower uptake of RIF NS is probably due to either the absence of active transport ligand or presence of a negative charge on its surface. Thus, RIF bearing NS was shown to be a promising candidate for lowering the drug toxicity against A549 cells.

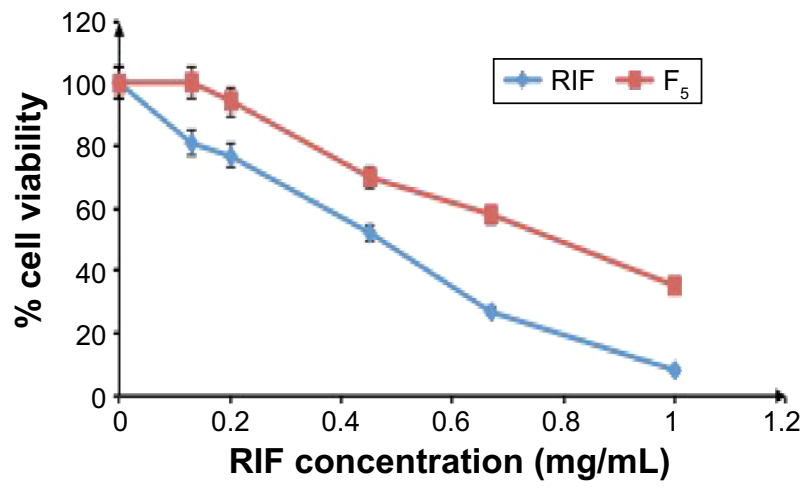

Figure 9 Percent viability of A549 cells measured by MTT cytotoxicity assay after exposure for 24 hours to various concentrations of either free RIF or RIF NS $\left(F_{5}\right)$ at $37 \pm 0.5^{\circ} \mathrm{C}$, mean $\pm S D(n=3)$.

Abbreviations: MTT, 3(4,5-dimethylthiazol-2-yl)-2,5-diphenyltetrazolium bromide; RIF, rifampicin; RIF NS, rifapmicin nanosuspension; SD, standard deviation.

\section{Conclusion}

The current study proved the possibility of RIF NS fabrication via a simple ultrasonication antisolvent precipitation technique. Nanocrystal-shaped particles were prepared with adequate nanometric particle size, PdI, and ZP. Solubility and in vitro release profiles of the RIF NS were significantly improved in comparison to the drug powder. Furthermore, it exhibited a good physical stability at $4 \pm 0.5^{\circ} \mathrm{C}$ within a period of 3 months. Moreover, RIF NS was able to reduce the cytotoxicity of the human alveolar epithelial cells compared to the free drug. Overall, fabrication of RIF NS not only, reveals promising in-vitro properties but also paves the way for an efficient strategy for a safe targeted delivery of such a poorly soluble drug to the lung.

\section{Disclosure}

The authors report no conflicts of interest in this work.

\section{References}

1. Mehanna MM, Mohyeldin SM, Elgindy NA. Respirable nanocarriers as a promising strategy for antitubercular drug delivery. J Control Release. 2014;187:183-197.

2. Caminero JA, Sotgiu G, Zumla A, Migliori GB. Best drug treatment for multidrug-resistant and extensively drug-resistant tuberculosis. Lancet Infect Dis. 2010;10:621-629.

3. Pandey R, Ahmad Z. Nanomedicine and experimental tuberculosis: facts, flaws, and future. Nanomedicine. 2011;7:259-272.

4. Gumbo T, Louie A, Deziel MR, et al. Concentration-dependent Mycobacterium tuberculosis killing and prevention of resistance by rifampin. Antimicrob Agents Chemother. 2007;51:3781-3788.

5. Becker C, Dressman JB, Junginger HE, et al. Biowaiver monographs for immediate release solid oral dosage forms: rifampicin. J Pharm Sci. 2009;98:2252-2267.

6. Mariappan T, Singh S. Positioning of rifampicin in the biopharmaceutics classification system (BCS). Clin Res Regul Aff. 2006;23:1-10.

7. Burman WJ, Gallicano K, Peloquin C. Comparative pharmacokinetics and pharmacodynamics of the rifamycin antibacterials. Clin Pharmacokinet. 2001;40:327-341.

8. Saraogi GK, Gupta P, Gupta UD, Jain NK, Agrawal GP. Gelatin nanocarriers as potential vectors for effective management of tuberculosis. Int J Pharm. 2010;385:143-149.

9. Booysen LL, Kalombo L, Brooks E, et al. In vivo/in vitro pharmacokinetic and pharmacodynamic study of spray-dried poly-(dl-lactic-coglycolic) acid nanoparticles encapsulating rifampicin and isoniazid. Int J Pharm. 2013;444:10-17.

10. Tewes F, Brillault J, Couet W, Olivier JC. Formulation of rifampicincyclodextrin complexes for lung nebulization. J Control Release. 2008; 129:93-99.

11. Son YJ, McConville JT. A new respirable form of rifampicin. Eur J Pharm Biopharm. 2011;78:366-376.

12. Rabinow BE. Nanosuspensions in drug delivery. Nat Rev Drug Discov. 2004;3:785-796.

13. Kesisoglou F, Panmai S, Wu Y. Nanosizing - oral formulation development and biopharmaceutical evaluation. Adv Drug Deliv Rev. 2007;59: 631-644.

14. Tian X, Li H, Zhang D, et al. Nanosuspension for parenteral delivery of a p-terphenyl derivative: preparation, characteristics and pharmacokinetic studies. Colloids Surf B Biointerfaces. 2013;108:29-33.

15. Freag MS, Elnaggar YS, Abdallah OY. Development of novel polymerstabilized diosmin nanosuspensions: in vitro appraisal and ex vivo permeation. Int J Pharm. 2013;454:462-471. 
16. Das S, Suresh PK. Nanosuspension: a new vehicle for the improvement of the delivery of drugs to the ocular surface. Application to amphotericin B. Nanomedicine. 2011;7:242-247.

17. Saindane NS, Pagar KP, Vavia PR. Nanosuspension based in situ gelling nasal spray of carvedilol: development, in vitro and in vivo characterization. AAPS PharmSciTech. 2013;14:189-199.

18. Sinha B, Müller RH, Möschwitzer JP. Bottom-up approaches for preparing drug nanocrystals: formulations and factors affecting particle size. Int J Pharm. 2013;453:126-141.

19. Xia D, Quan P, Piao H, et al. Preparation of stable nitrendipine nanosuspensions using the precipitation-ultrasonication method for enhancement of dissolution and oral bioavailability. Eur J Pharm Sci. 2010;40:325-334.

20. Kakran M, Sahoo NG, Li L, Judeh MA. Fabrication of quercetin nanoparticles by anti-solvent precipitation method for enhanced dissolution. Powder Technol. 2012;223:59-64.

21. Hao L, Wang X, Zhang D, et al. Studies on the preparation, characterization and pharmacokinetics of Amoitone B nanocrystals. Int J Pharm. 2012;433:157-164.

22. Zhang H, Hollis CP, Zhang Q, Li T. Preparation and antitumor study of camptothecin nanocrystals. Int J Pharm. 2011;415:293-300.

23. Higuchi J, Connors K. Phase solubility techniques. Adv Anal Chem Instrum. 1965;4:117-212.

24. Ambrus R, Kocbek P, Kristl J, Sibanc R, Rajkó R, Szabó-Révész P. Investigation of preparation parameters to improve the dissolution of poorly water-soluble meloxicam. Int J Pharm. 2009;381:153-159.

25. Liu D, Xu H, Tian B, et al. Fabrication of carvedilol nanosuspensions through the anti-solvent precipitation-ultrasonication method for the improvement of dissolution rate and oral bioavailability. AAPS PharmSciTech. 2012;13:295-304.

26. Gao Y, Li Z, Sun M, et al. Preparation and characterization of intravenously injectable curcumin nanosuspension. Drug Deliv. 2011;18: 131-142.

27. Verma S, Gokhale R, Burgess DJ. A comparative study of top-down and bottom-up approaches for the preparation of micro/nanosuspensions. Int J Pharm. 2009;380:216-222.

28. Dong Y, Ng WK, Shen S, Kim S, Tan RB. Preparation and characterization of spironolactone nanoparticles by antisolvent precipitation. Int J Pharm. 2009;375:84-88.

29. Koradia DK, Parikh HR. Dissolution enhancement of albendazole through nanocrystal formulation. J Pharm Bioallied Sci. 2012;4: S62-S63.

30. Ungaro F, d'Angelo I, Coletta C, et al. Dry powders based on PLGA nanoparticles for pulmonary delivery of antibiotics: modulation of encapsulation efficiency, release rate and lung deposition pattern by hydrophilic polymers. J Control Release. 2012;157:149-159.

31. Matteucci ME, Hotze MA, Johnston KP, et al. Drug nanoparticles by antisolvent precipitation: mixing energy versus surfactant stabilization. Langmuir. 2006;22:8951-8959.

32. Thorat AA, Dalvi SV. Liquid antisolvent precipitation and stabilization of nanoparticles of poorly water soluble drugs in aqueous suspensions: recent developments and future perspective. Chem Eng J. 2012;181-182:1-34.

33. Hu J, Ng WK, Dong Y, Shen S, Tan RB. Continuous and scalable process for water-redispersible nanoformulation of poorly aqueous soluble APIs by antisolvent precipitation and spray-drying. Int J Pharm. 2011;404:198-204.

International Journal of Nanomedicine

\section{Publish your work in this journal}

The International Journal of Nanomedicine is an international, peerreviewed journal focusing on the application of nanotechnology in diagnostics, therapeutics, and drug delivery systems throughout the biomedical field. This journal is indexed on PubMed Central,

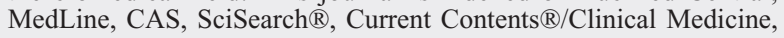

34. Wang Y, Zheng Y, Zhang L, Wang Q, Zhang D. Stability of nanosuspensions in drug delivery. J Control Release. 2013;172:1126-1141.

35. Verma S, Kumar S, Gokhale R, Burgess DJ. Physical stability of nanosuspensions: investigation of the role of stabilizers on Ostwald ripening. Int J Pharm. 2011;406:145-152.

36. Ali HS, York P, Ali AM, Blagden N. Hydrocortisone nanosuspensions for ophthalmic delivery: a comparative study between microfluidic nanoprecipitation and wet milling. J Control Release. 2011;149:175-181.

37. Sepassi S, Goodwin DJ, Drake AF, et al. Effect of polymer molecular weight on the production of drug nanoparticles. J Pharm Sci. 2007;96: 2655-2666.

38. Morakul B, Suksiriworapong J, Leanpolchareanchai J, Junyaprasert VB. Precipitation-lyophilization-homogenization (PLH) for preparation of clarithromycin nanocrystals: influencing factors on physicochemical properties and stability. Int J Pharm. 2013;457:187-196.

39. Ghosh I, Bose S, Vippagunta R, Harmon F. Nanosuspension for improving the bioavailability of a poorly soluble drug and screening of stabilizing agents to inhibit crystal growth. Int J Pharm. 2011;409:260-268.

40. Verma S, Huey BD, Burgess DJ. Scanning probe microscopy method for nanosuspension stabilizer selection. Langmuir. 2009;25: 12481-12487.

41. Choi J-Y, Yoo JY, Kwak H-S, Lee J. Role of polymeric stabilizers for drug nanocrystal dispersions. Curr Appl Phys. 2005;5:472-474.

42. Luque de Castro MD, Priego-Capote F. Ultrasound-assisted crystallization (sonocrystallization). Ultrason Sonochem. 2007;14:717-724.

43. Kakran M, Sahoo NG, Tan I-L, Li L. Preparation of nanoparticles of poorly water-soluble antioxidant curcumin by antisolvent precipitation methods. J Nanopart Res. 2012;14:1-11.

44. Guo J-j, Yue P-F, Lv J-1, et al. Development and in vivo/in vitro evaluation of novel herpetrione nanosuspension. Int J Pharm. 2013;441: 227-233.

45. Zu Y, Sun W, Zhao X, et al. Preparation and characterization of amorphous amphotericin B nanoparticles for oral administration through liquid antisolvent precipitation. Eur J Pharm Sci. 2014;53:109-117.

46. Zhang X, Xia Q, Gu N. Preparation of all-trans retinoic acid nanosuspensions using a modified precipitation method. Drug Dev Ind Pharm. 2006;32:857-863.

47. Dressman JB, Amidon GL, Reppas C, Shah VP. Dissolution testing as a prognostic tool for oral drug absorption: immediate release dosage forms. Pharm Res. 1998;15:11-22.

48. Agrawal S, Ashokraj Y, Bharatam PV, Pillai O, Panchagnula R. Solidstate characterization of rifampicin samples and its biopharmaceutic relevance. Eur J Pharm Sci. 2004;22:127-144.

49. Hong C, Dang Y, Lin G, et al. Effects of stabilizing agents on the development of myricetin nanosuspension and its characterization: an in vitro and in vivo evaluation. Int J Pharm. 2014;477:251-260.

50. Lefort R, De Gusseme A, Willart JF, Danède F, Descamps M. Solid state NMR and DSC methods for quantifying the amorphous content in solid dosage forms: an application to ball-milling of trehalose. Int JPharm. 2004; 280:209-219.

51. Dong S, HiraniI AA, Colacino KR, Lee YL, Roman M. Cytotoxicity and cellular uptake of cellulose nanocrystals. Nano LIFE. 2012;02: 1241006.

52. Mahmoud KA, Mena JA, Male KB, Hrapovic S, Kamen A, Luong JH. Effect of surface charge on the cellular uptake and cytotoxicity of fluorescent labeled cellulose nanocrystals. ACS Appl Mater Interfaces. 2010;2:2924-2932.

Journal Citation Reports/Science Edition, EMBase, Scopus and the Elsevier Bibliographic databases. The manuscript management system is completely online and includes a very quick and fair peer-review system, which is all easy to use. Visit http://www.dovepress.com/ testimonials.php to read real quotes from published authors. 